\title{
How to distinguish a nearly flat Universe from a flat Universe using the orientation independence of a comoving standard ruler
}

\author{
B. F. Roukema ${ }^{1,2 \star}$
}

\author{
1 Inter-University Centre for Astronomy and Astrophysics, Post Bag 4, Ganeshkhind, Pune, 411 007, India \\ 2 DARC/LUTH, Observatoire de Paris-Meudon, 5 place Jules Janssen, 92195 Meudon Cedex, France
}

Received 25 October 2000 / Accepted 30 January 2001

\begin{abstract}
Several recent observations using standard rulers and standard candles now suggest, either individually or in combination, that the Universe is close to flat, i.e. that the curvature radius is about as large as the horizon radius $\left(\sim 10 h^{-1} \mathrm{Gpc}\right)$ or larger. Here, a method of distinguishing an almost flat universe from a precisely flat universe using a single observational data set, without using any microwave background information, is presented. The method (i) assumes that a standard ruler should have no preferred orientation (radial versus tangential) to the observer, and (ii) requires that the (comoving) length of the standard ruler be known independently (e.g. from low redshift estimates). The claimed feature at fixed comoving length in the power spectrum of density perturbations, detected among quasars, Lyman break galaxies or other high redshift objects, would provide an adequate standard candle to prove that the Universe is curved, if indeed it is curved. For example, a combined intrinsic and measurement uncertainty of $1 \%$ in the length of the standard ruler $L$ applied at a redshift of $z=3$ would distinguish an hyperbolic $\left(\Omega_{\mathrm{m}}=0.2, \Omega_{\Lambda}=0.7\right)$ or a spherical $\left(\Omega_{\mathrm{m}}=0.4, \Omega_{\Lambda}=0.7\right)$ universe from a flat one to $1-P>95 \%$ confidence.
\end{abstract}

Key words. cosmology: observations - cosmology: theory - galaxies: clusters: general - large-scale structure of Universe - quasars: general

\section{Introduction}

In the Friedmann-Lemaître-Robertson-Walker model (Weinberg 1972), the Universe is an almost homogeneous 3 -manifold of constant curvature. This manifold may be the 3-hyperboloid $H^{3}$, flat Euclidean space $R^{3}$ or the 3 sphere $S^{3}$, or a quotient manifold of one of these, e.g. the 3-torus $T^{3}$ (Schwarzschild 1900, 1998).

A directly geometrical way to measure curvature is by a standard candle or a standard ruler, i.e. a class of objects of which the intrinsic brightness or comoving length scale is believed to be fixed. Several recent applications of standard candles or standard rulers include the standard candle applications of Perlmutter et al. (1999) and Riess et al. (1998), the standard ruler applications of Roukema \& Mamon (2000, 2001), Lange et al. (2000) and Balbi et al. (2000), which use geometrical information in the tangential direction, and the standard ruler application of Broadhurst \& Jaffe (2000), which uses geometrical information in the radial direction.

These recent measurements, individually or in combination, favour an "almost" flat local universe. However,

\footnotetext{
* e-mail: boud.roukema@obspm.fr
}

Jaffe et al. (2000) reject a flat universe to just under $95 \%$ significance, finding $\Omega_{\text {tot }}=1.11_{-0.12}^{+0.13}$ ("95\% confidence").

Whether the Jaffe et al. (2000) result is just due to random or systematic error and the observable Universe is in fact flat to high precision as predicted by many models of inflation, or whether the Universe really is measurably curved, distinguishing an almost (but not) flat universe from an "exactly" flat model will require considerably more precise techniques than have been previously applied.

Given the claimed existence of a comoving standard ruler (Broadhurst \& Jaffe 2000; Roukema \& Mamon 2000, 2001), what could possibly be the most model-free technique for testing the flatness hypothesis is presented here, for the case where the Universe is curved and independence of orientation of a comoving standard ruler is used to refute the flat universe hypothesis. Since

(i) a standard ruler should have no preferred orientation (radial versus tangential) with respect to the observer, and

(ii) the radial and tangential comoving distances differ by a sin or sinh factor if the Universe is curved (Eq. (5)),

then if the lengths of a standard ruler in the tangential and radial directions are proved to be unequal under the 
assumption of a flat universe, it might be thought that this would falsify the flat universe hypothesis. However, as shown below, the additional constraint that

(iii) the standard ruler should have a known, fixed comoving value is required in order to falsify the flat universe hypothesis.

Since the claimed standard ruler is in the linear regime of density perturbations, and is presumably a primordial feature in the power spectrum (if real), its inability to evolve in comoving length scale in less than a Hubble time would make it free of the evolutionary effects present for standard rulers or standard candles defined by collapsed objects.

Alcock \& Paczyñski (1979), Phillipps (1994), Matsubara \& Suto (1996) and Ballinger et al. (1996) have previously pointed out the potential usefulness of (i) and (ii), and have suggested applying these at quasi-linear or non-linear scales, i.e. at $r \lesssim 10 h^{-1} \mathrm{Mpc}$, including some analysis of how to try to separate out peculiar velocity effects. However, they did not discuss how to lift the degeneracy in the two curvature parameters $\left(\Omega_{\mathrm{m}}, \Omega_{\Lambda}\right)$ which remains after using (i) and (ii), and the problem of evolution of the $r \lesssim 10 h^{-1} \mathrm{Mpc}$ auto-correlation functions of galaxies and quasars offers potentially serious systematic uncertainties.

In contrast, by using a comoving standard ruler in the linear regime, the constancy in the comoving scale over a Hubble time [(iii) above] provides an additional constraint in the $\left(\Omega_{\mathrm{m}}, \Omega_{\Lambda}\right)$ plane, which is necessary in order to try to reject the flatness hypothesis. This is shown below (Fig. 1, Sect. 3).

Moreover, use of a linear regime comoving ruler also implies that peculiar velocity effects become negligible.

Note that since the method uses data from a single survey, it is qualitatively quite different from the concept of cosmic complementarity (Eisenstein et al. 1998; Lineweaver 1998).

The distance relations are reviewed in Sect. 2, a method of illustrating the principle is explained in Sect. 3, results are presented in Sect. 4, and conclusions are made in Sect. 5 .

\section{Distance relations}

Using the terminology of Weinberg (1972), the distance of use in the radial direction in comoving coordinates is the proper distance (Eq. (14.2.21), Weinberg 1972; denoted $R \chi$ by Peebles 1993, Eq. (13.28)),

$\mathrm{d}(z)=\frac{c}{H_{0}} \int_{1 /(1+z)}^{1} \frac{\mathrm{d} a}{a \sqrt{\Omega_{\mathrm{m}} / a-\Omega_{\kappa}+\Omega_{\Lambda} a^{2}}}$,

where $c$ is the speed of light, $H_{0}$ is the Hubble constant, $\Omega_{\mathrm{m}}$ is the matter density divided by the critical density, $\Omega_{\Lambda}$ is the cosmological constant, $z$ is redshift, and the dimensionless curvature is

$\Omega_{\kappa} \equiv \Omega_{\mathrm{m}}+\Omega_{\Lambda}-1$
The curvature radius is then

$R_{\mathrm{C}} \equiv \frac{c}{H_{0}} \frac{1}{\sqrt{\left|\Omega_{\kappa}\right|}}$.

For likely values of the curvature parameters, i.e. $\left(\Omega_{\mathrm{m}} \approx\right.$ $\left.0.3, \Omega_{\Lambda} \approx 0.7\right)$, the value of $R_{\mathrm{C}}$ is constrained to $R_{\mathrm{C}} \gtrsim$ $10 h^{-1}$ Gpc for a curvature of $\left|\Omega_{\kappa}\right| \lesssim 0$.1. Since the horizon radius, using proper distance $d$, is $R_{\mathrm{H}} \approx 10 h^{-1} \mathrm{Gpc}$, the present estimates of curvature can be succintly restated as

$R_{\mathrm{C}} \gtrsim R_{\mathrm{H}} \approx 10 h^{-1} \mathrm{Gpc}$.

The proper distance is not usually useful in observational cosmology, unless phenomena in comoving coordinates are being studied. The distances more commonly used are the proper motion distance (p. 485, Weinberg 1972; called "angular size distance" by Peebles 1993, p. 319, Eq. (13.29))

$d_{\mathrm{pm}}(z)=\left\{\begin{array}{ll}R_{\mathrm{C}} \sinh \left[d(z) / R_{\mathrm{C}}\right], & \Omega_{\kappa}<0 \\ d(z), & \Omega_{\kappa}=0 \\ R_{\mathrm{C}} \sin \left[d(z) / R_{\mathrm{C}}\right], & \Omega_{\kappa}>0\end{array}\right.$,

and distances which are greater or smaller than this by a factor of $(1+z)$.

The tangential distance of use in comoving coordinate work can be written as

$$
\begin{aligned}
d_{\perp}(z, \delta \theta) & \equiv \delta \theta d_{\mathrm{pm}}(z) \\
& =\delta \theta \begin{cases}R_{\mathrm{C}} \sinh \left[d(z) / R_{\mathrm{C}}\right], & \Omega_{\kappa}<0 \\
d(z), & \Omega_{\kappa}=0 \\
R_{\mathrm{C}} \sin \left[d(z) / R_{\mathrm{C}}\right], & \Omega_{\kappa}>0\end{cases}
\end{aligned}
$$

where $\delta \theta$ is an angle in radians on the sky. For "diagonal" comoving distances, thought of as arc-lengths by embedding comoving 3 -space in a four-dimensional space, see Roukema (2001).

\section{Method}

Equations (1) and (6) clearly show that for a standard ruler placed at a large fraction of the curvature radius from the observer, the sin or sinh term will strongly distinguish the curved and flat cases.

In order to test for independence of orientation of a standard ruler, consider a standard ruler of fixed comoving size $L$ which is observationally detected near a redshift $z$, radially as a redshift interval $\delta z$ and tangentially as an angular size $\delta \theta$. Using Eqs. (1) and (6), the length of the ruler placed in the radial direction is

$d\left(z+\delta z, \Omega_{\mathrm{m}}, \Omega_{\Lambda}\right)-d\left(z, \Omega_{\mathrm{m}}, \Omega_{\Lambda}\right)$

and the length in the tangential direction is

$d_{\perp}\left(z, \delta \theta, \Omega_{\mathrm{m}}, \Omega_{\Lambda}\right)$,

where the implicit dependence on the curvature parameters is now made explicit. Define the difference between these as

$\Delta d\left(z, \Omega_{\mathrm{m}}, \Omega_{\Lambda}\right) \equiv$

$d_{\perp}\left(z, \delta \theta, \Omega_{\mathrm{m}}, \Omega_{\Lambda}\right)-\left[d\left(z+\delta z, \Omega_{\mathrm{m}}, \Omega_{\Lambda}\right)-d\left(z, \Omega_{\mathrm{m}}, \Omega_{\Lambda}\right)\right]$. 
Unless the Copernican principle is very surprisingly violated, $\Delta d$ should be equal to zero, within the measurement uncertainties and intrinsic uncertainties of the ruler. If the Universe is "slightly" curved, then a flat universe can be refuted if

$\Delta d\left(z, \Omega_{\mathrm{m}}, \Omega_{\Lambda} \equiv 1-\Omega_{\mathrm{m}}\right)=0$

can be refuted for all acceptable values of $\Omega_{\mathrm{m}}$, or if the solutions to the equation are for values of $d_{\perp}$ inconsistent with $L$.

The method is then defined by

(a) choosing the acceptable range of $\Omega_{\mathrm{m}}$, e.g. $0 \leq \Omega_{\mathrm{m}} \leq 1$,

(b) choosing $z, L$ and $\Delta L$ (the uncertainty in $L$ ),

(c) calculating the implied values of $\delta z$ and $\delta \theta$,

(d) assuming $\Delta L$ to be the total measurement and intrinsic uncertainty (in length units) in each of the radial and tangential directions separately,

and then calculating

$\Delta d\left(z, \Omega_{\mathrm{m}}, 1-\Omega_{\mathrm{m}}\right)$

and the Gaussian probability that any of these values are consistent with zero, i.e. "orientation-free",

$P_{\mathrm{o}-\text { free }}=\operatorname{erfc}[|\Delta d| /(\sqrt{2} \sigma)]$,

where $\sigma^{2}=2(\Delta L)^{2}$, for each value of $\Omega_{\mathrm{m}}$.

As is seen in Fig. 1 and discussed in Sect. 4, this is insufficient on its own to rule out a flat universe hypothesis, so the additional hypothesis that

$d_{\perp}\left(z, \delta \theta, \Omega_{\mathrm{m}}, 1-\Omega_{\mathrm{m}}\right)=L$,

is required, where the Gaussian probability is

$P_{\mathrm{L}}=\operatorname{erfc}\left[\left|d_{\perp}\left(z, \delta \theta, \Omega_{\mathrm{m}}, 1-\Omega_{\mathrm{m}}\right)-L\right| /(\sqrt{2} \sigma)\right]$,

where $\sigma^{2}=2(\Delta L)^{2}$ as above. (The radial ruler size could equally well be used here.)

The independence of the ruler from orientation and the value of the length of the ruler are independent hypotheses, so the hypothesis of a flat universe is then rejected at the

$1-P_{\mathrm{o}-\text { free }} P_{\mathrm{L}}$

confidence level.

\section{Results}

Figure 1 shows that for reasonable values of $\left(\Omega_{\mathrm{m}}, \Omega_{\Lambda}\right)$ for a non-flat universe, the sin or sinh factor which relates the radial and tangential directions, making a ruler of different sizes if the universe is curved (Eq. (6)), can be compensated for by the non-linearity in the distance redshift relation. In other words, even if the Universe really is "slightly" curved, a wrong pair of values $\left(\Omega_{\mathrm{m}}, \Omega_{\Lambda}\right)$, where $\Omega_{\mathrm{m}}$ is larger (smaller) than the true value for a hyperbolic (spherical) universe, can be found for which the radial and

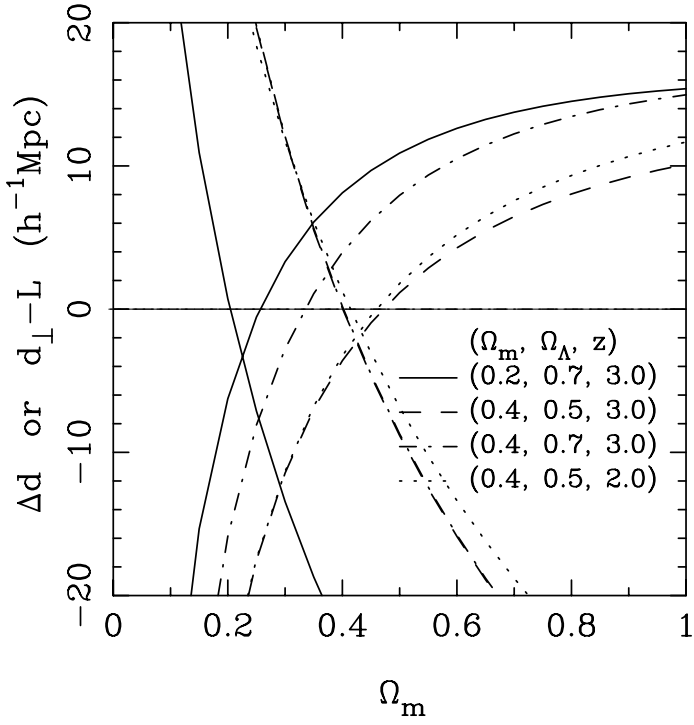

Fig. 1. Differences between tangential and radial lengths implied by assuming a flat universe, $\Delta d\left(z, \Omega_{\mathrm{m}}, 1-\Omega_{\mathrm{m}}\right)$ (Eq. (10)), and differences between the tangential ruler size and its known size, $d_{\perp}\left(z, \delta \theta, \Omega_{\mathrm{m}}, 1-\Omega_{\mathrm{m}}\right)-L$, both shown in comoving $h^{-1}$ Mpc as a function of $\Omega_{\mathrm{m}}$. The $\Delta d$ curves increase with $\Omega_{\mathrm{m}}$; the $d_{\perp}-L$ curves decrease with $\Omega_{\mathrm{m}}$. Various input hypotheses for hyperbolic and spherical universe models and redshifts $z$ are shown with different curves as labelled. The points where the increasing curves pass through zero are those where a flat universe implies independence of the ruler size from orientation. The points where the decreasing curves pass through zero are those where the ruler size is the known (e.g. zero redshift) size of the ruler

tangential sizes of the standard ruler are equal. These solutions are represented by the zero crossings of the curves which increase with $\Omega_{\mathrm{m}}$ in Fig. 1. The differences between the "true" $\Omega_{\mathrm{m}}$ values and those required for independence of $L$ from orientation (for a wrong, flat solution) are not large.

This is why the size of the ruler needs to be known (Eq. (13)). The curves of $d_{\perp}-L$ in Fig. 1 (which decrease with increasing $\Omega_{\mathrm{m}}$ ) are reasonably steep near both the input and the "orientation-free, flat" values of $\Omega_{\mathrm{m}}$, but to distinguish the point of intersection of the curves from zero would require uncertainties of much less than $10 h^{-1} \mathrm{Mpc}$, or $\ll 10 \%$ of the ruler size.

The probabilities for rejecting the flat universe based on the individual requirements of orientation independence (Eq. (12)), correctness of the value of $L$ (Eq. (14)) and the combined probabilities (Eq. (15)) are shown in Figs. 2 and 3. In the latter figure, higher precision, i.e. $1 \%$, is assumed in $L$. The minimum values of $1-P_{\mathrm{o}-\text { free }} P_{\mathrm{L}}$ are listed in Table 1.

For an uncertainty of $\sim 10 \%$, none of the "slightly" curved models, each having $\left|\Omega_{\kappa}\right|=0.1$ and $R_{\mathrm{C}} \approx R_{\mathrm{H}} \approx$ $10 h^{-1} \mathrm{Gpc}$, would enable significant rejection of a flat universe. However, for an uncertainty of $1 \%$, all three of the same "slightly" curved models, using data at $z=3$, would enable significant rejection of a flat universe, i.e. at the 


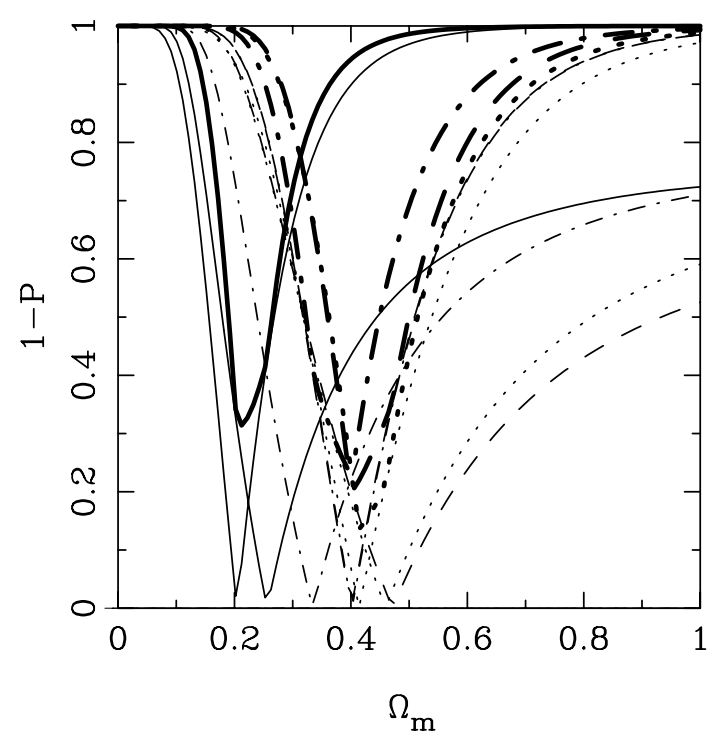

Fig. 2. Rejection probabilities based on the results in Fig. 1, assuming that $L=130 h^{-1} \mathrm{Mpc}, \Delta L=10 h^{-1} \mathrm{Mpc}$, and using Eqs. (12), (14) and (15). Curve styles are as for Fig. 1. The thin curves are for $1-P_{\mathrm{o}-\text { free }}$ and $1-P_{\mathrm{L}}$, the bold curves are for $1-P_{\mathrm{o}-\text { free }} P_{\mathrm{L}}$

$1-P \gtrsim 95 \%$ level. Lower redshift data $(z=2)$ only provides marginally significant rejection.

For the potentially most interesting case of $\Delta L=1 \% L$ and $z=3$, Table 2 shows how the strength of the rejection varies with $\Omega_{\mathrm{m}}$ and $\Omega_{\kappa}$. A nearly flat universe with a value of the matter density considerably higher than presently estimated, i.e. $\Omega_{\mathrm{m}} \geq 0.5$, would be more difficult to reject, given a fixed absolute curvature $\left|\Omega_{\kappa}\right|=0.1$. On the other hand, a larger absolute curvature $\left(\left|\Omega_{\kappa}\right|=0.2\right)$ would enable refutation of a flat universe to better than $99.7 \%$ confidence for $\Omega_{\mathrm{m}} \leq 0.5$.

\section{Discussion and conclusions}

Does a standard ruler of the required precision exist? This depends, of course, on what the curvature of the Universe really is. If the Universe is "exactly" flat in a geometrical sense, i.e. if the covering space is $R^{3}$, then use of local geometrical techniques would not be sufficient to prove that the Universe is not curved. Proof that the Universe is flat and multiply connected (e.g. see Luminet \& Roukema 1999 for a review) would be one way of using global geometry to prove that the Universe is not (on average) curved.

However, if the radius of curvature is no bigger than the horizon, then the calculations above show that for reasonable values of $\left(\Omega_{\mathrm{m}}, \Omega_{\Lambda}\right)$, a precision of $1 \%$ in the application of a standard ruler at a redshift of $z=3$ would be sufficient. At higher redshifts, less precision would be needed, but the possibility of having large surveys including sufficient amounts of both radial and tangential standard ruler information is unlikely in the next decade at $z \gg 3$.

At $z=3$, surveys of quasars or of Lyman break galaxies (e.g. Adelberger et al. 1998; Giavalisco et al. 1998)

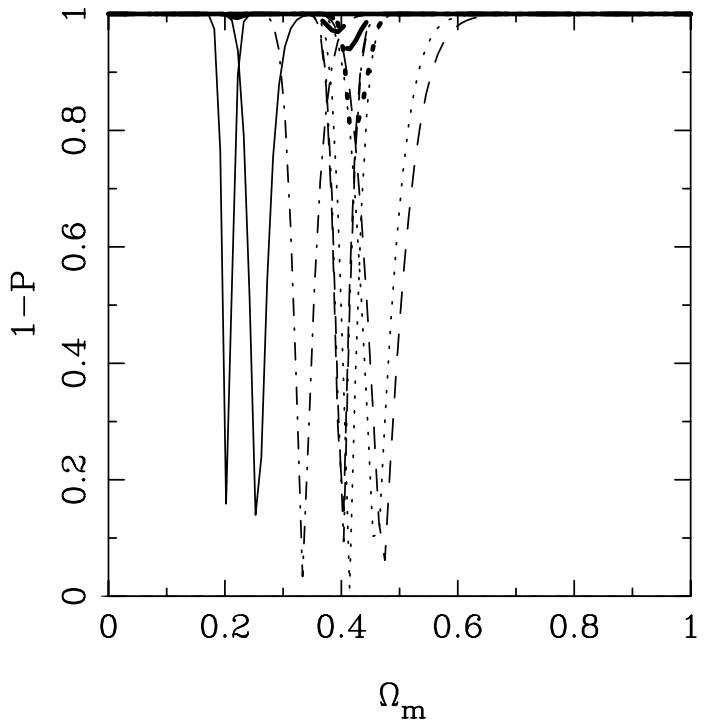

Fig. 3. As for Fig. 2, for $L=130 h^{-1} \mathrm{Mpc}, \Delta L=1.3 h^{-1} \mathrm{Mpc}$ (i.e. $1 \%$ precision). The $1-P_{\mathrm{o}-\text { free }} P_{\mathrm{L}}$ curves in bold are barely visible, close to the $100 \%$ rejection limit. Their minima are listed in Table 1

of sufficient quality to detect comoving features at large scales in the power spectrum should be feasible.

Whether or not fixed, comoving features in the power spectrum of density perturbations, as traced by these objects, exist and are detectable, is still a controversial subject. Observations by several different groups suggest that a peak near the maximum in the power spectrum, at $L \approx 130 \pm 10 h^{-1} \mathrm{Mpc}$, is common to galaxies and superclusters of galaxies at low redshift (Broadhurst et al. 1990; Broadhurst \& Jaffe 2000; da Costa et al. 1993; Baugh \& Efstathiou 1993; Gaztañaga \& Baugh 1998; Einasto et al. 1994, 1997; Deng et al. 1996; Guzzo 1999; Tucker et al. 1998) and Lyman break objects (Broadhurst \& Jaffe 2000) and quasars (Deng et al. 1994; Roukema \& Mamon $2000,2001)$ at high redshift. Since $130 h^{-1} \mathrm{Mpc}$ is above the present turnaround scale, it should be fixed in comoving coordinates. Moreover, several possible theoretical explanations for this feature, which would also imply other "oscillations" in the power spectrum, include acoustic oscillations in the baryon-photon fluid before last scattering, in high baryon density models (Eisenstein et al. 1998; Meiksin et al. 1998; Peebles 1999), and features from sub-Planck length physics which survive through to oscillations in the post-inflation power spectrum, for weakly coupled scalar field driven inflationary models (Martin \& Brandenberger 2000a,b).

No group yet claims that the precision of the peak is better than $10 \%$. Observational improvements (homogeneity of surveys, numbers of objects), refinements in statistical analysis techniques, and an unambiguous theoretical explanation for the peak might help reduce the uncertainty in the value.

What prospects for observational improvements from surveys expected to be completed within $1-5$ years might potentially approach $1 \%$ precision in $L$ ? 
Table 1. Minimum rejection values $1-P_{\mathrm{o}-\text { free }} P_{\mathrm{L}}$ of the bold curves in Figs. 2 and 3

\begin{tabular}{ccccccc}
\hline$\Omega_{\mathrm{m}}$ & $\Omega_{\Lambda}$ & $\Omega_{\kappa}$ & $\begin{array}{c}z \\
\left(h^{-1} \mathrm{Mpc}\right)\end{array}$ & $\begin{array}{c}L \\
\left(h^{-1} \mathrm{Mpc}\right)\end{array}$ & $\Delta L$ & $\min \left\{1-P_{\mathrm{o}-\text { free }} P_{\mathrm{L}}\right\}$ \\
\hline 0.2 & 0.7 & -0.1 & 3 & 130 & 10 & $31 \%$ \\
0.4 & 0.5 & -0.1 & 3 & 130 & 10 & $20 \%$ \\
0.4 & 0.7 & +0.1 & 3 & 130 & 10 & $24 \%$ \\
0.4 & 0.5 & -0.1 & 2 & 130 & 10 & $14 \%$ \\
0.2 & 0.7 & -0.1 & 3 & 130 & 1.3 & $99.7 \%$ \\
0.4 & 0.5 & -0.1 & 3 & 130 & 1.3 & $94 \%$ \\
0.4 & 0.7 & +0.1 & 3 & 130 & 1.3 & $97 \%$ \\
0.4 & 0.5 & -0.1 & 2 & 130 & 1.3 & $80 \%$ \\
\hline
\end{tabular}

Table 2. Minimum rejection values $1-P_{\mathrm{o}-\text { free }} P_{\mathrm{L}}$, as for Table 1 , but for a larger range in $\Omega_{\mathrm{m}}, \Omega_{\Lambda}$, with $z=3, L=130 h^{-1} \mathrm{Mpc}$, $\Delta L=1.3 h^{-1} \mathrm{Mpc}$ fixed

\begin{tabular}{cccccccc}
\hline$\Omega_{\mathrm{m}}$ & $\Omega_{\Lambda}$ & $\Omega_{\kappa}$ & $\min \left\{1-P_{\mathrm{o}-\text { free }} P_{\mathrm{L}}\right\}$ & $\Omega_{\mathrm{m}}$ & $\Omega_{\Lambda}$ & $\Omega_{\kappa}$ & $\min \left\{1-P_{\mathrm{o}-\text { free }} P_{\mathrm{L}}\right\}$ \\
\hline \multicolumn{6}{c}{ dependence on $\Omega_{\mathrm{m}}$} \\
0.1 & 0.8 & -0.1 & $100.0 \%$ & 0.1 & 1.0 & 0.1 & $100.0 \%$ \\
0.2 & 0.7 & -0.1 & $99.7 \%$ & 0.2 & 0.9 & 0.1 & $99.9 \%$ \\
0.3 & 0.6 & -0.1 & $97.6 \%$ & 0.3 & 0.8 & 0.1 & $99.2 \%$ \\
0.4 & 0.5 & -0.1 & $93.9 \%$ & 0.4 & 0.7 & 0.1 & $97.2 \%$ \\
0.5 & 0.4 & -0.1 & $89.9 \%$ & 0.5 & 0.6 & 0.1 & $93.8 \%$ \\
0.6 & 0.3 & -0.1 & $86.4 \%$ & 0.6 & 0.5 & 0.1 & $89.5 \%$ \\
0.7 & 0.2 & -0.1 & $83.4 \%$ & 0.7 & 0.4 & 0.1 & $84.9 \%$ \\
0.8 & 0.1 & -0.1 & $77.5 \%$ & 0.8 & 0.3 & 0.1 & $80.1 \%$ \\
0.9 & 0.0 & -0.1 & $70.5 \%$ & 0.9 & 0.2 & 0.1 & $75.3 \%$ \\
& & & $\mathrm{a}$ higher value of $\left|\Omega_{\kappa}\right|$ & & \\
0.1 & 0.7 & -0.2 & $100.0 \%$ & 0.1 & 1.1 & 0.2 & $100.0 \%$ \\
0.2 & 0.6 & -0.2 & $100.0 \%$ & 0.2 & 1.0 & 0.2 & $100.0 \%$ \\
0.3 & 0.5 & -0.2 & $100.0 \%$ & 0.3 & 0.9 & 0.2 & $100.0 \%$ \\
0.4 & 0.4 & -0.2 & $99.9 \%$ & 0.4 & 0.8 & 0.2 & $100.0 \%$ \\
0.5 & 0.3 & -0.2 & $99.7 \%$ & 0.5 & 0.7 & 0.2 & $100.0 \%$ \\
0.6 & 0.2 & -0.2 & $99.2 \%$ & 0.6 & 0.6 & 0.2 & $99.9 \%$ \\
0.7 & 0.1 & -0.2 & $98.3 \%$ & 0.7 & 0.5 & 0.2 & $99.6 \%$ \\
0.8 & 0.0 & -0.2 & $97.2 \%$ & 0.8 & 0.4 & 0.2 & $99.0 \%$ \\
0.9 & -0.1 & -0.2 & $95.6 \%$ & 0.9 & 0.3 & 0.2 & $98.0 \%$ \\
\hline
\end{tabular}

Table 3. Statistical redshift accuracy $\Delta z$ needed for positions of low redshift structures used for calibrating $L$. For a given redshift $z$ and given metric parameters, the uncertainty in redshift $\Delta z$ corresponding to $\Delta L=1.3 h^{-1} \mathrm{Mpc}(1 \%$ precision in $\left.L=130 h^{-1} \mathrm{Mpc}\right)$ is given. This shows that over the domain shown, a precision of $\Delta z \lesssim 510^{-4}$ in redshift is required

\begin{tabular}{ccccc}
\hline$\Omega_{\mathrm{m}}$ & $\Omega_{\Lambda}$ & \multicolumn{3}{c}{$\mathrm{z}$} \\
& & 0.1 & 0.3 & 0.5 \\
\hline 0.3 & 0.0 & 0.0005 & 0.0006 & 0.0007 \\
0.3 & 0.7 & 0.0005 & 0.0005 & 0.0006 \\
1.0 & 0.0 & 0.0005 & 0.0006 & 0.0008 \\
\hline
\end{tabular}

The best possible improvements in precision would presumably scale as Poisson errors, so that improving from $\sim 10 \%$ precision to $1 \%$ would require a factor of 100 increase in the numbers of objects relative to previous surveys.

The continuation of the original observations by Broadhurst et al. (1990) which confirm the original result include more than 1000 spectroscopic redshifts up to maximum redshifts of $z \sim 0.4$ (Broadhurst \& Jaffe 2000; Broadhurst 1999). The VIRMOS shallow survey (Le Fèvre et al. 2001) is expected to obtain 100000 spectroscopic redshifts of galaxies in fields of size $\sim 2^{\circ}$ to a limiting magnitude of $I_{\mathrm{AB}}=22.5$, i.e. with typical median (maximum) redshifts of $z \sim 0.6(z \sim 1.3)$ (Crampton et al. 1995). This is probably the best near future survey which more or less matches the conditions of the Broadhurst et al. (1990) observations. As long as the fact that the galaxies will be spread over a somewhat larger redshift range does not adversely affect improvements in the estimation of $L$, 
the factor of $\sim 100$ increase in numbers should be sufficient to provide the precision required.

However, if the effect is anisotropic as suggested by Einasto et al. (1997), then the VIRMOS shallow survey may not be enough, since none of the four fields are close to the directions of the Broadhurst et al. fields.

Wide angle surveys may therefore be more useful.

In the 2dF Galaxy Redshift Survey (2dFGRS, Colless et al. 1999) it is expected to observe spectroscopic redshifts of 250000 galaxies over 2000 sq deg with a mean redshift of $z=0.1$. For the Sloan Digital Sky Survey (SDSS, Sloan et al. 2001), it is planned to observe about 1000000 galaxy spectroscopic redshifts over $10000 \mathrm{sq}$ deg with a median redshift of $z=0.1$.

Both surveys clearly provide the increase in numbers of objects required. A possible problem in precision is the fact that only about 100 galaxies/sq deg will be observed in these surveys as opposed to around 1000 galaxies/sq deg in the Broadhurst et al. (1990) fields. If individual structures ("walls", "filaments") are less sharply traced in position relative to the Broadhurst et al. (1990) surveys, then this could provide an additional noise factor.

Will spectroscopic redshift accuracy be sufficient not to provide an additional source of uncertainty in the radial direction? Table 3 shows that a precision in redshift of $\Delta z \lesssim 510^{-4}$ corresponds to $\Delta L=1.3 h^{-1} \mathrm{Mpc}$.

The VIRMOS survey (Le Fèvre et al. 2001) is only expected to achieve spectral resolution of $\lambda / \Delta \lambda \sim 250$ for the full 100000 galaxies, implying $\Delta z \sim 410^{-3}(1+$ $z) \lesssim 10^{-2}$. Although a sub-sample will be observed at $\lambda / \Delta \lambda \sim 2500-5000$ in order to study biases, it is hard to see how this can be sufficient for the purposes of obtaining a precise value of $L$. It would be preferable if the full 100000 galaxy sample could be observed at the higher resolution.

On the other hand, the spectral resolutions of the $2 \mathrm{dF}$ GRS and SDSS are expected to be $\lambda / \Delta \lambda \sim 1000-2000$, so will provide approximately the precision required.

However, $\Delta z \lesssim 510^{-4}$ precision in redshift corresponds to $\Delta z c \lesssim 300 \mathrm{kms}^{-1}$. Typical galaxy velocity dispersions in loose groups and clusters and typical bulk velocities, or in other words, typical galaxy velocities with respect to the comoving reference frame, are typically at about this scale or up to nearly an order of magnitude higher. So, averaging over these "random" errors will be required in order to obtain the precision required.

In the analysis of the low redshift surveys, it should be kept in mind that even though the redshifts are smaller than unity, a clear and precise detection of the $L$ scale would require approximately correct values of the metric parameters, and may be missed if wrong values are used.

It should also be noted that, in the hypothesis that the proposed ruler actually exists, and can be traced back to the primordial Universe, the theoretical explanation of the scale might heavily rely on some assumption on yet unknown or difficult to measure cosmological or fundamental physical parameters. This would introduce an additional uncertainty if the theoretical model were to be used to define the size of the standard ruler.

In conclusion, if the Universe is indeed "slightly" curved, then this method could potentially be promising for proving that the Universe is not flat.

Note that small-scale clustering on quasi-linear or nonlinear scales (cf. Alcock \& Paczyñski 1979) would be more difficult to use as a standard ruler, due to evolution in the ruler length.

Acknowledgements. The author thanks Gary Mamon, Stéphane Colombi, Tarun Deep Saini, Varun Sahni and Thanu Padmanabhan, whose comments were useful and encouraging. The support of the Institut d'Astrophysique de Paris, CNRS, and of DARC, Observatoire de Paris-Meudon, for visits during which part of this work was carried out, and the support of la Société de Secours des Amis des Sciences are gratefully acknowledged.

\section{References}

Adelberger, K. L., Steidel, C. C., Giavalisco, M., et al. 1998, ApJ, 508, 18

Alcock, C., \& Paczyñski, B. 1979, Nature, 281, 358

Balbi, A., Ade, P., Bock, J., et al. 2000, ApJL, in press, [arXiv: astro-ph/0005124]

Ballinger, W. E., Peacock, J. A., \& Heavens, A. F. 1996, MNRAS, 282, 877

Baugh, C. M., \& Efstathiou, G. 1993, MNRAS, 265, 145

Broadhurst, T. 1999, oral presentation at the "Clustering at High Redshift" IGRAP meeting, Marseille, 29 June-2 July 1999

Broadhurst, T. J., Ellis, R. S., Koo, D. C., \& Szalay, A. S. 1990, Nature, 343, 726

Broadhurst, T., \& Jaffe, A. H. 2000, ApJL, in press, [arXiv: astro-ph/9904348]

Colless, M. M. 1999, in Looking Deep in the Southern Sky, ed. R. Morganti, \& W. J. Couch, ESO Astrophysics Symposia (Springer), 9; and 2dFGRS team,

http://www.mso.anu.edu.au/2dFGRS/

Crampton, D., Le Fevre, O., Lilly, S. J., \& Hammer, F. 1995, ApJ, 455, 96

da Costa, L. N., et al. 1993, in Cosmic Velocity Fields, ed. F. Bouchet, \& M. Lachièze-Rey (Gif-sur-Yvette, France: Éditions Frontières), 475

Deng, X.-F., Deng, Z.-G., \& Xia, X.-Y. 1996, Chin. Astron. Astroph., 20, 383

Deng, Z., Xia, X.-Y., \& Fang, L.-Zh. 1994, ApJ, 431, 506

Einasto, M., Einasto, J., Tago, E., Dalton, G. B., \& Andernach, H. 1994, MNRAS, 269, 301

Einasto, J., Einasto, M., Gottloeber, S., et al. 1997, Nature, 385,139

Eisenstein, D. J., Hu, W., Silk, J., \& Szalay, A. S. 1998, ApJ, 494, L1

Eisenstein, D. J., Hu, W., \& Tegmark, M. 1998, ApJ, 504, L57

Gaztañaga, E., \& Baugh, C. M. 1998, MNRAS, 294, 229

Giavalisco, M., Steidel, C. C., Adelberger, K. L., et al. 1998, ApJ, 503, 543 [arXiv:astro-ph/9802318]

Guzzo, L. 1999, in proceedings of XIX Texas Symp. Rel. Astr. [arXiv: astro-ph/9911115]

Jaffe, A. H., et al. 2000, preprint [arXiv:astro-ph/0007333] 
Lange, A. E., Ade, P. A. R., Bock, J. J., et al. 2000, preprint [arXiv:astro-ph/0005004]

Le Fèvre, O., et al. 2001, VIRMOS Team, http://www. astrsp-mrs.fr/virmos/

Lineweaver, C. H. 1998, ApJ, 505, L69

Luminet, J.-P., \& Roukema, B. F. 1999, in Theoretical and Observational Cosmology, NATO Advanced Study Institute, Cargèse 1998, ed. M. Lachièze-Rey, (Dordrecht, Netherlands: Kluwer), 117 [arXiv:astro-ph/9901364]

Martin, J., \& Brandenberger, R. H. 2000a, preprint [arXiv:hep-th/0005209]

Martin, J., \& Brandenberger, R. H. 2000b, preprint [arXiv : astro-ph/0005432]

Meiksin, A., White, M., \& Peacock, J. A. 1999, MNRAS, 304, 851 [arXiv:astro-ph/9812214]

Matsubara, T., Suto, Y. 1996, ApJ, 470, L1

Peebles, P. J. E. 1993, Principles of Physical Cosmology (Princeton, USA: Princeton Univ. Press)

Peebles, P. J. E. 1999, ApJ, 510, 531
Perlmutter, S., Aldering, G., Goldhaber, G., et al. 1999, ApJ, 517, 565 [arXiv:astro-ph/9812133]

Phillipps, S. 1994, MNRAS, 269, 1077

Riess, A. G., et al. 1998, AJ, 116, 1009

Roukema, B. F. 2001, MNRAS, in press [arXiv: astro-ph/0102099]

Roukema, B. F., \& Mamon, G. A. 2000, A\&A, 358, 395 [arXiv:astro-ph/9911413]

Roukema, B. F., \& Mamon, G. A. 2001, A\&A, 366, 1 [arXiv: astro-ph/0010511]

Schwarzschild, K. 1900, Vier. d. Astr. Gess., 35, 337

Schwarzschild, K. 1998, Class. Quant. Grav., 15, 2539

Sloan, A. P., et al. 2001, Sloan Digital Sky Survey Team, http://www.sdss.org/science/tech_summary.html

Tucker, D. L., Lin, H., \& Shectman, S. 1998, in Wide Field Surveys in Cosmology, ed. S. Colombi, Y. Mellier, \& B. Raban [arXiv:astro-ph/9902023]

Weinberg, S. 1972, Gravit. Cosmol. (New York, USA: Wiley) 